\title{
Urinary obstruction from sexual practice involving magnetized beads inserted in the male urethra
}

\author{
Tyler Brooks MD, Jamie Zreick BSc, Angelo locca MD
}

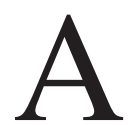
26-year-old man presented to clinic with a 3-day history of worsening dysuria and decreased urinary output; he had no additional systemic symptoms. He reluctantly disclosed that on the evening before the onset of symptoms, his girlfriend had inserted beads into his urethra as part of their sexual activity that night. The patient did not know the number or type of beads used.

On physical examination, the patient was not in distress, his vital signs were normal, and his penis and abdomen were unremarkable. The results of urinalysis were normal. Urine culture was ordered, as were tests for chlamydia and gonorrhea. Because only distal urethral obstruction was considered based on the initial history, a radiograph of the penis was ordered; the radiograph showed no radioopaque foreign bodies.

The patient was discharged after being given antibiotic coverage for urinary tract infection, including possible chlamydia and gonorrhea infection, while awaiting test results. The patient was instructed to keep up with oral fluid intake and to return to the clinic the following day if there was no improvement.

The following day, the patient returned to the clinic unable to void, despite increased oral fluid intake. The patient had subsequently learned that the beads that had been inserted into his urethra were $1 / 4$ inch spherical, metallic and magnetized beads. After counting them at home, he and his partner discovered that 42 of the beads were missing.

A radiograph of his pelvis showed multiple beads in his bladder (Figure 1). The patient was sent to the emergency department of the local hospital for urgent cystoscopy. The urologist used a basket and "three-pronged grasper" to retrieve the beads one at a time via the urethra. The retrieval process took about 1.5 hours. The patient's recovery was uneventful. Possible future complications include urethral stricture.

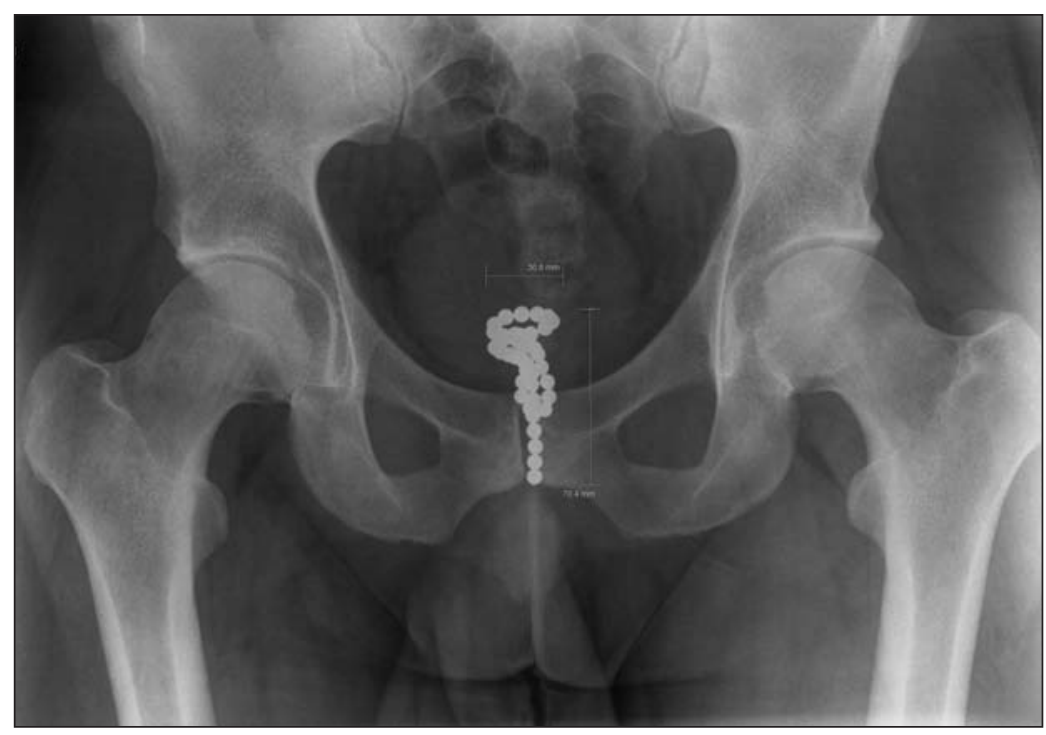

Figure 1: Radiograph of the patient's pelvis, showing multiple metallic beads in his bladder.

\section{Discussion}

Male urethral stimulation is the sexual practice of inserting objects into the distal urethra for the purpose of enhancing sensory response during sexual activity. It has been described as the most common nonmedical reason for the insertion of foreign objects into the male urethra. ${ }^{1}$ This practice may produce a pleasurable stimulatory effect because of the common innervation of both the urethra and glans penis by the dorsal nerve of the penis, a connection that has been
Competing interests: None declared.

This article has been peer reviewed.

Correspondence to:

Tyler Brooks,

tyler_brooks@tricolour.que ensu.ca

CMAJ 2013. DOI:10.1503 /cmaj.130397

\section{- Ker pOINTS}

- Male urethral stimulation is a sexual practice wherein objects, including medical devices, purpose-built "sex toys" or improvised devices, are inserted into the urethra for sexual stimulation.

- There has been a recent cluster of case reports of the urethral insertion of magnetized metal beads, leading to urinary symptoms.

- Clinicians should consider the possibility of foreign body obstruction for patients who present with lower urinary tract symptoms.

- Foreign bodies may be introduced as far as the urinary bladder. 
shown by electrodiagnostic studies involving human volunteers. ${ }^{2}$ Inserted objects may be medical devices, such as sounds or probes, purpose-built commercial "sex toys," such as plugs or rods, or improvised objects not intended for urethral insertion. Devices specifically designed for urethral stimulation often have an enlarged end or handle to prevent their loss within the urethra. However, improvised devices may lack such features, risking migration of the object and inability to withdraw it from the urethra. In the case described here, the objects ascended as far as the urinary bladder.

This case of magnetized steel balls becoming lodged in the urinary bladder after insertion in the urethra is not unique. ${ }^{3}$ Three nearly identical cases have been reported recently in Canada. ${ }^{4}$ These cases differed only in the treatment choice of open cystotomy rather than minimally invasive cystoscopy to successfully remove the balls. The powerful, rare-earth magnet balls were described as "beads that are found in a toy store." ${ }^{4}$ Clearly, these were improvised objects used for urethral insertion.

This recent cluster of similar reported cases may be instructive from a clinical medicine perspective. Indeed, they may signal some changes in overall sexual attitudes, particularly when taken in the context of other social trends, such as the mainstream popularity of books that describe sexual practices perhaps previously regarded as appealing only to a minority of the population. The coincidence of these cases and trends in entertainment may be suggestive of a broader societal interest and participation in sexually adventurous activities, such as male urethral stimulation. Clinicians, therefore, need to consider the possibility of foreign object obstruction in the context of lower urinary tract symptoms.

\section{References}

1. Stravodimos KG. Electrical wire as a foreign body in a male urethra: a case report. J Med Case Rep 2009;3:49.

2. Yang CC, Bradley WE. Innervation of the human anterior urethra by the dorsal nerve of the penis. Muscle Nerve 1998;4: 514-8.

3. Graziottin TM, Soares DD, Da Ros CT, et al. Magnetic spheres as foreign body into the bladder. J Sex Med 2012 May 21 [Epub ahead of print].

4. Levine MA, Evans H. Open removal as a first-line treatment of magnetic intravesical foreign bodies. Can Urol Assoc J 2013; 7:E25-8.

Affiliations: Primary care physician (Brooks), physician assistant (Zreick), Barrie, Ont., Division of Urology, Royal Victoria Regional Health Centre (Iocca), Barrie, Ont.

Contributors: All of the authors conceived and drafted the manuscript. All of the authors revised the manuscript for important intellectual content and approved the final version submitted for publication. 